\title{
Healing of cutaneous wounds using GaAs laser in diabetic rat models
}

\author{
P. Nekha Krishnan', P. T. Dinesh ${ }^{2}$, S. Sooryadas ${ }^{3}$, Reji Varghese ${ }^{4}$ and M. Pradeep ${ }^{5}$ \\ Department Of Veterinary Surgery and Radiology, \\ College of Veterinary and Animal Sciences, Pookode, Wayanad- 673576 \\ Kerala Veterinary and Animal Sciences University, Kerala, India.
}

Citation: Nekha, K.P., Dinesh, P.T., Sooryadas, S., Varghese, R. and Pradeep, M. 2021. Healing of cutaneous wounds using GaAs laser in diabetic rat models. J. Vet. Anim. Sci. 52(3): 234-237.

DOI: https://doi.org/10.51966/jvas.2021.52.3.234-237

Received: 14.01.2021

Accepted:27.02.2021

Published: 30.09 .2021

\begin{abstract}
Conventional treatment modalities usually fail to heal wounds in diabetic animals. Photomodulation of the wound bed using low-level laser has proved its efficacy in treating chronic wounds. Considering the fact that the majority of the diabetic wounds end up in amputation of affected part and reduce the quality of life of the patient, the present study was undertaken to find out the efficacy of gallium arsenide (GaAs) laser in the healing of full-thickness cutaneous wounds in type 2 diabetic rat models. The study was conducted in six adult male Wistar rats with an average body weight of 150 $g$ in which hyperglycemia coexisting with hyperlipidemia was induced. Two wounds of $1 \mathrm{~cm}^{2}$ area were induced of which left side wounds served as the control and right side wounds were treated with $\mathrm{GaAs}$ laser at $4 \mathrm{~J} / \mathrm{cm}^{2}$ area for one minute continuously for four days.
\end{abstract}

Keywords: Type 2 diabetes, streptozotocin, GaAs laser

Changes in the way of life of human beings have led to the development of a variety of lifestyle diseases. Since in modern times pets are like family members, the situation applies to them also. Wounds in diabetic patients are difficult to heal due to diabetic neuropathy and degraded growth factors. Management of such wounds require unconventional or modern treatment modalities so that healing of the wounds can happen fast. Low-level laser therapy (LLLT) involves the use of a laser of wavelengths between 500 and $1100 \mathrm{~nm}$ and typically involves the delivery of $1-4 \mathrm{~J} / \mathrm{cm}^{2}$ to the treatment site having output power between 10 and $90 \mathrm{~mW}$ (Hawkins et al., 2005). Photomodulation of the wound bed with low energy laser has been proved efficient in bringing about healing of the chronic wounds. (Varghese, 2002). Irradiation of the wound bed using low level

1. M.V.Sc. scholar

2. Assistant Professor and Head, Email: dineshpt@kvasu.ac.in Ph : 9447144085

3. Assistant Professor

4. Assistant Professor, TVCC, Mannuthy

5. Assistant Professor, Department of Veterinary Pathology

Copyright: () 2021 Nekha et al. This is an open access article distributed under the terms of the Creative Commons Attribution 4.0 International License (http://creativecommons.org/licenses/by/4.0/), which permits unrestricted use, distribution, and reproduction in any medium, provided the original author and source are credited. 
laser at appropriate doses cause stimulation of cell function, producing biological, chemical and physical effects without thermal effects (Posten et al., 2005) This in turn promotes wound healing.

In diabetic wounds, infection and inflammation leads to imbalance of protease and reactive oxygen species, degradation of essential growth factors, the ability of angiogenesis is impaired and cell recruitment to the wound sites is inhibited. This in turn is found to delay wound healing. Considering the above, a study was conducted to find out the efficacy of gallium arsenide (GaAs) laser for the management of wounds in type II diabetic rat models.

\section{Materials and methods}

The study was approved by the Institutional Animal Ethics Committee of College of Veterinary and Animal Sciences, Pookode of Kerala Veterinary and Animal Sciences University (IAEC/ COVAS/ PKD/16/2019 dtd. 17/01/2019).

Ten adult male Wistar rats with an average body weight of $150 \mathrm{~g}$ were randomly selected for the study. Hyperlipidemia was induced by feeding a high-fat diet for three weeks as per Suman et al. (2016) in all the rats. The high-fat diet was prepared by mixing standard rat diet, raw cholesterol, and a mixture of vanaspati and coconut oil in the ratio of 2:1. Two per cent of raw cholesterol powder was mixed in coconut oil and was also fed orally at the dose rate of $3 \mathrm{~mL} / \mathrm{kg}$ body weight. Hyperlipidemia was confirmed by estimating serum total cholesterol levels after three weeks. Diabetes mellitus was induced by a single dose of streptozotocin at the rate of $40 \mathrm{mg} / \mathrm{kg}$ body weight given intraperitoneally. Diabetes mellitus was confirmed by estimating blood glucose levels after three days. Two wounds of size $1 \mathrm{~cm}^{2}$ area each were created on either side of the body in the thoracolumbar region under general anaesthesia. The wound bed was irradiated with GaAs laser at $4 \mathrm{~J} / \mathrm{cm}^{2}$ for 1 minute for four consecutive days followed by dressing with povidone-iodine lotion till the end of the observation period. Control wounds were treated with povidone-iodine lotion until the end of the study. Observations were continued till both the wounds healed completely.

Healing of the wound was assessed by monitoring the factors as suggested by Reddy et al. (2012) which included nature of wound edges, presence of necrotic tissue, nature of exudate if present, tissue oedema and induration, amount, colour and texture of granulation tissue and epithelialisation. Wound surface area was estimated by the method put forth by Jothi et al. (2007) in which the wound area was plotted on a graph paper and counted. The rate of wound contraction was estimated by the formula put forth by Kirubanandan and Sehgal (2010)

\section{Results and discussion}

Hyperlipidemia could be induced with the feeding protocol adopted in this experiment. The total cholesterol value ranged from 123 - $278 \mathrm{mg} / \mathrm{dL}$ with a mean of $181.57 \pm 20.09$. The high cholesterol level was maintained throughout the period of observation. Diabetes mellitus was effectively induced with a single intraperitoneal injection of streptozotocin at the rate of $40 \mathrm{mg} / \mathrm{kg}$ body weight. The serum glucose levels ranged from 203- $584 \mathrm{mg} / \mathrm{dL}$ with a mean of $358.29 \pm 18.25$. The high glucose level was maintained throughout the period of observation.

According to Grey et al. (2006) the edges of a healthy healing wound slops towards the centre whereas that of a non-healing wound will be either punched out or rolled out in nature. In the present study, both the treated and the control wounds showed a sloping wound edge which indicated that the wound was healing.

In the present study, the condition of the wound was assessed by monitoring the colour of the wound bed. As per Grey et al (2006 wound could be considered healthy and healing when the wound bed is pink in colour whereas unhealthy if the wound bed is dark red or maroon. All the wounds in the treatment site remained pink in colour indicating that the wounds were healthy and healing whereas the control wounds were dark red during the initial days of observation indicating an unhealthy initial phase. 
Grey et al., (2006) suggested that the amount of granulation tissue present in the wound during the healing phase determines the healing rate. It should be moderate to medium in a healing wound. The wound will not heal if the quantity of granulation tissue is minimum and can become a Keloid if it is heavy. In the present study, the amount of granulation tissue in the treated wounds were moderate to medium whereas, it was minimum in control wounds. Similar findings were observed by Lazovic et al. (2005).

Epithelialisation and generation of epithelium over a wound is the final stage of healing. Epithelial tissue which is light pink in colour, migrates inwards from the wound edges or may appear as small islands of tissue over the surface of the wound (Fraser, 2020). The treated wounds in the present study started epithelisation by day five but was minimal except in rat 8 in which it was moderate. In rats 21,17 and 18 maximum epithelisation could be noticed by day 9 . In control wounds, epithelisation started by day seven, which was minimum. Similar observations were made by Lavozic et al. (2005), Mitra et al. (2015), and Karri et al. (2016).

The wounds were mapped onto a sterile transparent sheet and the area was estimated by graphical method. The wound surface area $\left(\mathrm{cm}^{2}\right)$ is presented in table 1 . Wound surface area was statistically analysed using one-way ANOVA The wound surface area did not differ significantly between the control and treatment groups on day zero. The surface area showed significant $(p<0.05)$ difference between the control and treatment groups during the subsequent days of observation and it was highly significant $(p<0.01)$ on days five and seven.

The rate of wound contraction was estimated using the formula $\left(\left(A_{0}-A_{n}\right) / A_{0}\right) \times 100$ where $A_{0}$ is the wound area in day zero and $A_{n}$ is the wound area in $\mathrm{n}^{\text {th }}$ day.

On day three, the mean rate of wound contraction (\%) on control side was $17.34 \pm 5.07$,

\begin{tabular}{|l|c|c|c|c|}
\hline & Day 0 & Day 3 & Day 7 & Day 11 \\
\hline & & & \\
\hline \\
\hline
\end{tabular}

Fig.1. Gross morphology of wounds

Table 1. Wound Surface area $\left(\mathrm{cm}^{2}\right) \mathrm{n}=10$

\begin{tabular}{|l|c|c|c|c|c|}
\hline & Day 0 & Day 3 & Day 5 & Day 7 & Day 9 \\
\hline Control Wound & $0.97 \pm 0.38$ & $0.79 \pm 0.03$ & $0.48 \pm 0.06$ & $0.20 \pm 0.04$ & $0.05 \pm 0.01$ \\
\hline Treatment Wound & $1.04 \pm 0.02$ & $0.68 \pm 0.09$ & $0.31 \pm 0.06$ & $0.13 \pm 0.03$ & $0.01 \pm 0$ \\
\hline T value & $1.39^{\text {ns }}$ & $1.21^{\text {ns }}$ & $2.28^{*}$ & $2.90^{*}$ & $4.34^{* *}$ \\
\hline P value & 0.22 & 0.31 & 0.09 & 0.03 & 0.007 \\
\hline \multicolumn{7}{|c|}{ ns-non-significant at 0.05 level; ${ }^{*}$ - significant at 0.05 level; ${ }^{* *}$ - significant at 0.01 level }
\end{tabular}


and on the treatment side it was $24.22 \pm 8.05$ On day five, the rate of wound contraction was $49.37 \pm 7.67,55.43 \pm 5.11$, in control and treated wounds, respectively. The rate of wound contraction on day seven was $78.55 \pm 5.59$ for the control wounds and $86.69 \pm 3.79$ for the treated wounds. The rate of wound contraction (\%) on day nine of control wounds was $94.17 \pm 2.03$ and that of treated wounds was $98.19 \pm 1.25$

The average time of complete healing of treated wounds was $8.8 \pm 0.33$ days and on control wounds, it was $10.4 \pm 0.40$ days $(p<0.01)$.

\section{Conclusion}

Based on the present study the wound treated with GaAs laser took lesser time to heal compared to that of the control wound. Wounds treated with GaAs laser healed comparatively faster than the control wound. The size of the treatment wound significantly reduced from the day 5. Laser treated wound completely healed on day 9. However, the control wound healed completely only after 12 days.

\section{Conflict of interest}

The authors declare that they have no conflict of interest.

\section{References}

Fraser, B., 2020 The colour of wounds and its implication for healing. Available at http://healthtimes.com.au/hub/ wound-care/59/practice/bfl/the-colourof-wounds-and-its-implication-forhealing/45/[13 October 2020]

Grey, J. E., Enoch, S. and Harding, K. G. 2006. ABC of wound healing: wound assessment. Br. Med. J. Practice. 332: 285-288.

Hawkins, D., Houreld, N. and Abrahamse, H., 2005. Low level laser therapy (LLLT) as an effective therapeutic modality for delayed wound healing.

Ann. N. Y. Acad. Sci. 1056: 486-493.

Jothi, N. A., Thilagar, S., Omar, A. R. S., Kamaruddin, M. D., Shanthi, G., Goh, Y. M. and Sabri, M. Y. 2007. Effects of biomaterials keratin-gelatin and basic fibroblast growth factor-gelatin composite film on wound healing in dogs. J. Vet. Malaysia 18: 21-26.

Karri, V.V.S.R., Kuppusamy, G., Talluria, S.V., Mannemala, S.S., Kollipara, R., Wadhwani, A.D., Mulukutla, S., Raju, K.R.S. and Malayandi, R. 2016. Curcumin loaded chitosan nanoparticles impregnated into collagen-alginate scaffolds for diabetic wound healing. Int. J. Biol. Macromolec. 93:1519-1529.

Kirubanandan, S. and Sehgal, P. 2010. Regeneration of soft tissue using porous bovine collagen scaffold. J. Optoelectr. Biomed. Mat. 2: 141-149.

Lazovic, G., Colic, M., Grubor, M. and Jovanovic, M., 2005. The application of collagen sheet in open wound healing. Annals of burns and fire disasters, 18:151.

Mitra, T., Manna, P. J., Raja, S. T. K., Gnanamani, A. and Kundu, P. P. 2015. Curcumin loaded nano graphene oxide reinforced fish scale collagen-a 3D scaffold biomaterial for wound healing applications. RSC Adv. 5: 98653-98665.

Posten, W., Wrone, D. A., Dover, J. S., Arndt, K. A., Silapunt, S. and Alam, M. 2005. Lowlevel laser therapy for wound healing: mechanism and efficacy. Dermatol. Surg. 31: 334-340.

Reddy, M., Gill, S. S., Wu, W., Kalkar, S. R. and Rochon, P. A. 2012. Does this patient have an infection of a chronic wound? J. Am. Med. Assoc. 307: 605-611.

Suman, R. K., Mohantey, I. R, Bordey, M. K., Maheswari, U. and Deshmukh, Y. A. 2016. Development of an experimental model of diabetes co-existing with metabolic syndrome in rats. $A d v$ Pharmacol. Sci. Available: http//dx.doi. org/10.1155/2016/9463476 05/01/2018

Varghese, R. 2002. Studies on the effect of Helium-Neon laser on healing of chronic wounds in dogs. MVSc thesis, Tamilnadu Veterinary and Animal Sciences University, Chennai, 65p. 